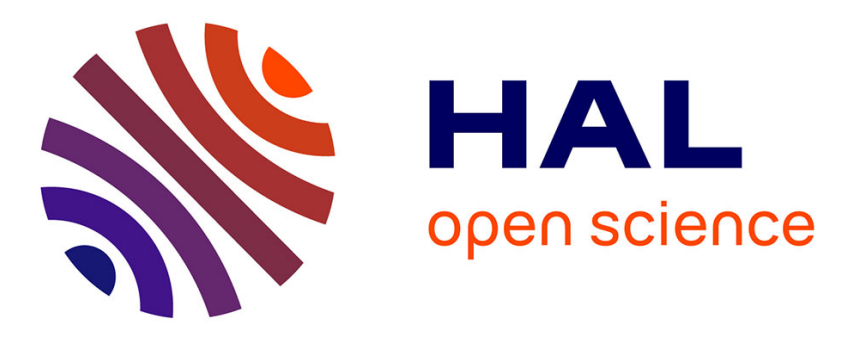

\title{
Second-Order Blind Source Separation: A New Expression of Instantaneous Separating Matrix for Mixtures of Delayed Sources
}

Gilles Chabriel-, Jean Barrère

\section{- To cite this version:}

Gilles Chabriel-, Jean Barrère. Second-Order Blind Source Separation: A New Expression of Instantaneous Separating Matrix for Mixtures of Delayed Sources. 2006 IEEE 7th Workshop on Signal Processing Advances in Wireless Communications , Jul 2006, Cannes, France. pp.1 - 5, 10.1109/SPAWC.2006.346397 . hal-01427439

\section{HAL Id: hal-01427439 \\ https://hal-univ-tln.archives-ouvertes.fr/hal-01427439}

Submitted on 5 Jan 2017

HAL is a multi-disciplinary open access archive for the deposit and dissemination of scientific research documents, whether they are published or not. The documents may come from teaching and research institutions in France or abroad, or from public or private research centers.
L'archive ouverte pluridisciplinaire HAL, est destinée au dépôt et à la diffusion de documents scientifiques de niveau recherche, publiés ou non, émanant des établissements d'enseignement et de recherche français ou étrangers, des laboratoires publics ou privés. 


\title{
SECOND-ORDER BLIND SOURCE SEPARATION: A NEW EXPRESSION OF INSTANTANEOUS SEPARATING MATRIX FOR MIXTURES OF DELAYED SOURCES
}

\author{
Gilles Chabriel - Jean Barrère \\ STIC-TOULON/GESSY \\ Université du Sud Toulon-Var \\ Av. de l'Université, BP 20132 \\ 83162 LA GARDE Cedex (FRANCE) \\ Fax: (33) 494142598 \\ chabrieleuniv-tln.fr - barrereeuniv-tln.fr
}

\begin{abstract}
In this paper, we study the blind separation of mixtures of propagating waves (delayed sources) encountered for example in underwater telephone (UWT) systems . We suggest a new second-order statistics method using as many observations as sources. First, we show that each of the $N$ delayed sources can be developed as a particular linear combination of the different temporal-derivatives of the $N$ observations. Under some assumptions, an instantaneous rectangular separating matrix is then identified by the joint diagonalization of a set of covariance matrices estimated from the observations and its derivatives.

The algorithm used takes into account the particular structure of the spectral mixing matrix encountered.

A numerical simulation is provided in a 3-sources/3- observations case for propagating audio signals.
\end{abstract}

\section{INTRODUCTION}

Consider a set of $N$ propagating waves $s_{j}(t)$ in an echo-free and noise-free medium. These are recorded on a set of $M$ identical sensors. Let $x_{j}(t)$ denote the contribution of the source $s_{j}(t)$ on a sensor arbitrary indexed by 1 , i.e. $x_{j}(t)$ is a filtered version of the source $s_{j}(t)$. Assuming that the set of sensors is sufficiently compact, then the contribution of the source $s_{j}(t)$ recorded on a sensor $i$ is the same one as that recorded on the first sensor, except for an attenuation factor and a propagation delay. The observation $y_{i}(t)$ recorded on the sensor $i$, is a linear combination of the delayed contribu-

$$
\begin{aligned}
\text { tions } x_{j}(t) \text { : } & \begin{aligned}
y_{1}(t) & =\sum_{j=1}^{N} x_{j}(t), \\
y_{i}(t) & =\sum_{j=1}^{N} c_{i j} x_{j}\left(t-\tau_{i j}\right), \quad i \in[2, \ldots, M],
\end{aligned}
\end{aligned}
$$

where $c_{i j}$ is the relative attenuation coefficient of source $j$ on sensor $i$, and $\tau_{i j}$ a relative propagation delay of source $j$ on sensor $i$.

The different contributions are assumed to be band-limited, differently colored, statistically independent and zero-mean. Thereafter in the paper, the contributions will be called sources.

The problem is to provide an estimation of the $N$ different sources $x_{j}(t)$ from the observations $y_{i}(t)$.

A first way to solve the problem using truncated Taylor series of each delayed source $x_{j}\left(t-\tau_{i j}\right)$ has been successfully treated in [1]-[5]. It consists of the estimate of an instantaneous square mixing matrix $\mathbf{M}_{M \times M}$ between the observations and the different derivatives of the sources:

$$
\left[y_{1}, y_{2}, \ldots\right]^{T}=\mathbf{M}\left[x_{1}, x_{2}, \ldots, \dot{x}_{1}, \dot{x}_{2}, \ldots\right]^{T} .
$$

For low delays, second-order statistics are sufficient to extract the different contributions from the observations (see [2], [4], [6]). For higher delays, a combination of two-order and highorder statistics methods is proposed in [3] to achieve the separation.

The main limitation of these methods is the high necessary number of observations (up to several times the number of sources).

We propose here a $N$-sources $N$-sensors original approach based on the direct estimation of an instantaneous rectangular 
separating matrix $\mathbf{S}$ between the different derivatives of the observations and filtered sources:

$\left[x_{1}^{h}, x_{2}^{h}, \ldots x_{N}^{h},\right]^{T}=\mathbf{S}\left[y_{1}, \dot{y}_{1}, \ldots, y_{2}, \dot{y}_{2}, \ldots, y_{N}, \dot{y}_{N}, \ldots\right]^{T}$ where $x_{j}^{h}$ is the source $x_{j}$ filtered by an invertible known filter $h$.

It is important to note that the two approaches are not equivalent: the matrix $\mathbf{M}$ is not a simple pseudoinverse of $\mathbf{S}$.

The paper is organized as follows: in the next section we explain how to construct the separating matrix $\mathbf{S}$ from the propagation model described by the system of equations (1). Then the third section explains the second-order statistics method implemented to estimate this separating matrix. The estimation uses a new version of the joint-diagonalization algorithm proposed by S.Dégerine in [7]. A description of this algorithm has to appear in english, a preprint can already be read in [8]. A numerical simulation in the last section illustrates the effectiveness of our approach.

\section{EXPRESSION OF THE SEPARATING MATRIX}

In the frequency domain, the system (1) becomes

$$
\begin{aligned}
Y_{1}(\nu) & =\sum_{j=1}^{N} X_{j}(\nu), \\
Y_{i}(\nu) & =\sum_{j=1}^{N} c_{i j} e^{-\jmath 2 \pi \nu \tau_{i j}} X_{j}(\nu), \quad i \in[2, \ldots, N] .
\end{aligned}
$$

$X_{j}(\nu)$ and $Y_{i}(\nu)$ are respectively the Fourier Transforms (FT) of the $j^{\text {th }}$ source and of the $i^{\text {th }}$ observation.

The system (2) can be rewritten in matrix notation as:

$$
\mathbf{Y}(\nu)=\mathbf{M}^{f}(\nu) \mathbf{X}(\nu),
$$

where

$\mathbf{Y}(\nu)=\left[Y_{1}(\nu), \ldots, Y_{N}(\nu)\right]^{T}, \mathbf{X}(\nu)=\left[X_{1}(\nu), \ldots, X_{N}(\nu)\right]^{T}$.

The $N \times N$ spectral mixing matrix $\mathbf{M}^{f}(\nu)$ is defined as:

$$
\mathbf{M}^{f}(\nu)=\left[\begin{array}{ccc}
1 & \ldots & 1 \\
c_{21} e^{-\jmath 2 \pi \nu \tau_{21}} & \ldots & c_{2 N} e^{-\jmath 2 \pi \nu \tau_{2 N}} \\
\vdots & \ddots & \vdots \\
c_{n 1} e^{-\jmath 2 \pi \nu \tau_{N 1}} & \ldots & c_{N N} e^{-\jmath 2 \pi \nu \tau_{N N}}
\end{array}\right] .
$$

We assume that $\mathbf{M}^{f}(\nu)$ is regular for any frequency. The inverse matrix of $\mathbf{M}^{f}(\nu)$ is

$$
\mathbf{M}^{f}(\nu)^{-1}=\frac{1}{\operatorname{det} \mathbf{M}^{f}(\nu)}\left(\operatorname{adj} \mathbf{M}^{f}(\nu)\right)^{T},
$$

where $\operatorname{adj} \mathbf{M}^{f}(\nu)$ and $\operatorname{det} \mathbf{M}^{f}(\nu)$ are respectively is the adjoint matrix and the determinant of the matrix $\mathbf{M}^{f}(\nu)$.

From equation (3), one has:

$$
\operatorname{det} \mathbf{M}^{f}(\nu) \mathbf{X}(\nu)=\left(\operatorname{adj} \mathbf{M}^{f}(\nu)\right)^{T} \mathbf{Y}(\nu) .
$$

According to the particular structure of the spectral mixing matrix $\mathbf{M}^{f}(\nu)$, its determinant (denoted by $H_{d}(\nu)$ ) is a sum of weighted complex exponentials:

$$
H_{d}(\nu)=\operatorname{det} \mathbf{M}^{f}(\nu)=\sum_{i} \beta_{i} \exp \left(-\jmath 2 \pi \nu \tau_{d_{i}}\right),
$$

where the weights $\beta_{i}$ are particular products of coefficients $c_{i j}$, and $\tau_{d_{i}}$ are particular sums of delays $\tau_{i j}$.

Let $\mathbf{H}(\nu)$ denote the transposed adjoint of $\mathbf{M}(\nu)$. Each entries $H_{k l}(\nu)$ of $\mathbf{H}(\nu)$ can also be expressed as a sum of weighted exponentials:

$$
H_{k l}(\nu)=\left(\operatorname{adj} \mathbf{M}^{f}(\nu)\right)_{k l}^{T}=\sum_{i} \alpha_{k l i} \exp \left(-\jmath 2 \pi \nu \tau_{k l i}\right),
$$

where the weights $\alpha_{k l i}$ are also particular products of coefficients $c_{i j}$, and $\tau_{k l i}$ are particular sums of delays $\tau_{i j}$.

Back to the temporal representation, the system (5) becomes:

$$
\left\{h_{d} * x_{k}\right\}(t)=\sum_{l=1}^{N}\left\{h_{k l} * y_{l}\right\}(t),
$$

where $h_{k l}(t)=\mathrm{FT}^{-1}\left(H_{k l}(\nu)\right), h_{d}(t)=\mathrm{FT}^{-1}\left(H_{d}(\nu)\right)$ and where $\{h . * x\}.(t)=\int h .(t-\tau) x .(\tau) d \tau$ denotes the convolution of $h .(t)$ and $x .(t)$.

Each filtered source $\left\{h_{d} * x_{k}\right\}(t)$ is then a finite sum of delayed observations:

$$
\left\{h_{d} * x_{k}\right\}(t)=\sum_{l, i} \alpha_{k l i} y_{l}\left(t-\tau_{k l i}\right) .
$$

Assuming that the $\tau_{k l i}$ are "small" for all indices, $y_{l}\left(t-\tau_{k l i}\right)$ can be approximated by its truncated up to P-order Taylor series expansion. Each filtered source can be then approximated by a linear combination of the observations and its derivatives:

$$
\left\{h_{d} * x_{k}\right\}(t) \approx \sum_{l, i} \alpha_{k l i} \sum_{p=0}^{P} \frac{\left(-\tau_{k l i}\right)^{p}}{p !} y_{l}^{(p)}(t) .
$$

For $k=1, \ldots, N$ the system is rewritten as:

$$
\mathbf{x}^{h}(t) \approx \mathbf{S} \tilde{\mathbf{y}}(t)
$$

where $\mathbf{x}^{h}(t)=\left[x_{1}^{h}(t), \ldots, x_{N}^{h}(t)\right]^{T}$ is the filtered sources vector, with $x_{k}^{h}(t)=\left\{h_{d} * x_{k}\right\}(t)$, 
$\tilde{\mathbf{y}}(t)=\left[y_{1}, \dot{y}_{1}, \ldots, y_{1}^{(P)}, y_{2}, \ldots, y_{2}^{(P)}, \ldots, y_{N}^{(P)}\right]^{T}$ is the observations and their derivatives vector. $\mathbf{S}$ is the $N \times(N P+N)$ separating matrix.

The analytical expression of each entry of $\mathbf{S}$ with respect to the parameters $c_{i j}$ and $\tau_{i j}$ is heavy but does not present theoretical difficulties. This expression is detailed in the Appendix A for the 2-sources/2-sensors case.

The joint estimation of the parameters $c_{i j}, \tau_{i j}$ and of the separating matrix $\mathbf{S}$ forms the basis of the iterative algorithm proposed in the following section.

\section{PRESENTATION OF THE ALGORITHM}

From (7) and because the sources $x_{k}^{h}(t)$ are mutually uncorrelated, it follows that $\mathbf{S}$ has to diagonalize the set of covariance matrices obtained at different lags $\mathbf{R}_{\tilde{\mathbf{y}} \tilde{\mathbf{y}}}\left(\tau_{k}\right)=\mathrm{E}\left\{\tilde{\mathbf{y}}(t) \tilde{\mathbf{y}}^{T}(t+\right.$ $\left.\left.\tau_{k}\right)\right\}$ :

$$
\mathbf{S R}_{\tilde{\mathbf{y}} \tilde{\mathbf{y}}}\left(\tau_{k}\right) \mathbf{S}^{T}=\boldsymbol{\Lambda}\left(\tau_{k}\right), \quad \forall \tau_{k}
$$

$\boldsymbol{\Lambda}\left(\tau_{k}\right)=\mathrm{E}\left\{\mathbf{x}^{h}(t) \mathbf{x}^{h^{T}}\left(t+\tau_{k}\right)\right\}$ being a diagonal matrix whatever the lag $\tau_{k}$

S. Dégerine propose in [7],[8] a new algorithm (called Least Square on B or LSB) for approximate non-orthogonal joint diagonalization of a set of matrices. In its original form, this algorithm iteratively searches an optimal joint diagonalizer $\tilde{\mathbf{S}}$ of a set of $K$ matrices to the mean square sense. The secondorder criterion to be optimized is then

$$
C(\tilde{\mathbf{S}})=\sum_{k} \operatorname{Off}\left(\tilde{\mathbf{S}} \mathbf{R}_{\tilde{\mathbf{y}} \tilde{\mathbf{y}}}\left(\tau_{k}\right) \tilde{\mathbf{S}}^{T}\right),
$$

where $O f f($.$) is the sum of the square non diagonal elements$ of the considered matrix. To perform the mean square optimization, S. Dégerine uses a relaxation on the lines of $\tilde{\mathbf{S}}$ conducting to solve $N$ eigenvalue problems for each iteration.

Here, the expected diagonalizer $\mathbf{S}$ being non square, the optimization (8) does not conduct to an unique solution for $\tilde{\mathbf{S}}$. The main idea is then to force the algorithm to hold the theoretical structure of the separation matrix at each iteration. This theoretical expression depends on the parameters $c_{i j}$ and $\tau_{i j}$. At each step of relaxation, we estimate the parameters $c_{i j}$ and $\tau_{i j}$ from their formal expression wrt the entries of the current estimated $\tilde{\mathbf{S}}$. The next step is processed using an new $\tilde{\mathbf{S}}$ built on these estimated parameters.

An illustration of such theoretical expressions can be found in Appendices for the 2-sources case. For the $N$-sources case, we implemented formal calculus subroutine in order to provide automatically the formal expressions we need.

The final algorithm is summed up as follows:

\section{Numerical Algorithm}

1) From the observations $\mathbf{y}(t)$, build the observation vector $\tilde{\mathbf{y}}(t)$ at the order $P$.

2) Compute $K$ covariance matrix $\mathbf{R}_{\tilde{\mathbf{y}} \tilde{\mathbf{y}}}\left(\tau_{k}\right)$.

3) Intitialize $\tilde{\mathbf{S}}(0)$ with arbitrary values.

4) Proceed to an iteration $k$ with LSB algorithm.

5) From $\tilde{\mathbf{S}}(k)$ obtained at step 4), estimate the attenuation coefficients $c_{i j}^{k}$ and the propagation delays $\tau_{i j}^{k}$.

6) From $c_{i j}^{k}$ and $\tau_{i j}^{k}$, compute a new matrix $\tilde{\mathbf{S}}(k+1)$.

7) Repeat steps 4), 5) and 6) until the evolution of the estimated matrix $\tilde{\mathbf{S}}$ becomes sufficiently weak.

\section{RESULTS}

We present now a numerical simulation in the 3-sources/3observations case. The sources are $24 \mathrm{~s}$ long music extracts. The sampling frequency is $F_{s}=22.05 \mathrm{kHz}$.

The matrix $\mathbf{C}$ of relative attenuations is:

$$
\mathbf{C}=\left[\begin{array}{lll}
1.0000 & 1.0000 & 1.0000 \\
2.0000 & 1.3000 & 1.2000 \\
3.0000 & 1.6000 & 2.2000
\end{array}\right]
$$

The matrix $\mathbf{D}$ of relative propagation delays is:

$$
\mathbf{D}=\left[\begin{array}{ccc}
0 & 0 & 0 \\
30.00 \mu s & 18.00 \mu s & 5.00 \mu s \\
25.00 \mu s & 12.50 \mu s & 10.00 \mu s
\end{array}\right]
$$

For the 3 observations, we take a Taylor series expansion up to 3 -order $(P=3)$. 151 covariance matrices $\mathbf{R}_{\tilde{\mathbf{y}} \tilde{\mathbf{y}}}\left(\tau_{k}\right)$ are used $(K=151)$, with $\tau_{k}=k / F s, k \in\{-75,75\}$.

Fig. 1 (a) shows the evolution of the optimization criterion $C(\tilde{\mathbf{S}})$ at each iteration number $i$ and part (b) presents the Frobenius norm of the matrix $\tilde{\mathbf{S}}(k+1)-\tilde{\mathbf{S}}(k)$ 

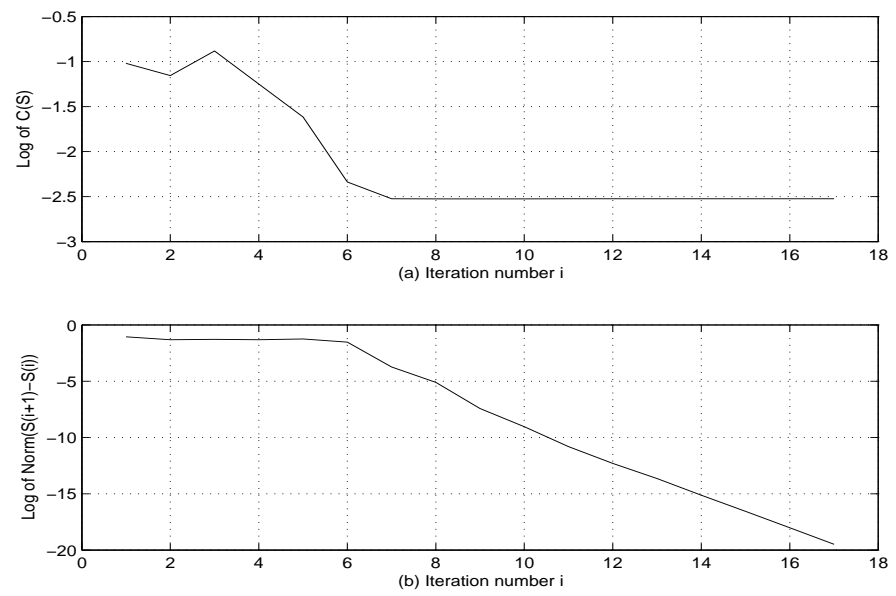

Fig. 1. Convergence

After convergence ( 8 iteration steps), we obtain the following estimations for the relative attenuations and for the relative propagation delays:

$$
\begin{gathered}
\tilde{\mathbf{C}}=\left[\begin{array}{lll}
1.0000 & 1.0000 & 1.0000 \\
1.9995 & 1.3047 & 1.1967 \\
2.9998 & 1.6053 & 2.1929
\end{array}\right], \\
\tilde{\mathbf{D}}=\left[\begin{array}{ccc}
0 & 0 & 0 \\
31.64 \mu s & 18.87 \mu s & 5.03 \mu s \\
26.56 \mu s & 13.13 \mu s & 9.81 \mu s
\end{array}\right] .
\end{gathered}
$$

Taking the matrices $\tilde{\mathbf{C}}$ and $\tilde{\mathbf{D}}$ into account, the filter $H_{d}(\nu)$ can be identified and its effect reversed after separation. We obtain the following source estimations (Fig. 2) that have to be compared to the original ones (Fig. 3).
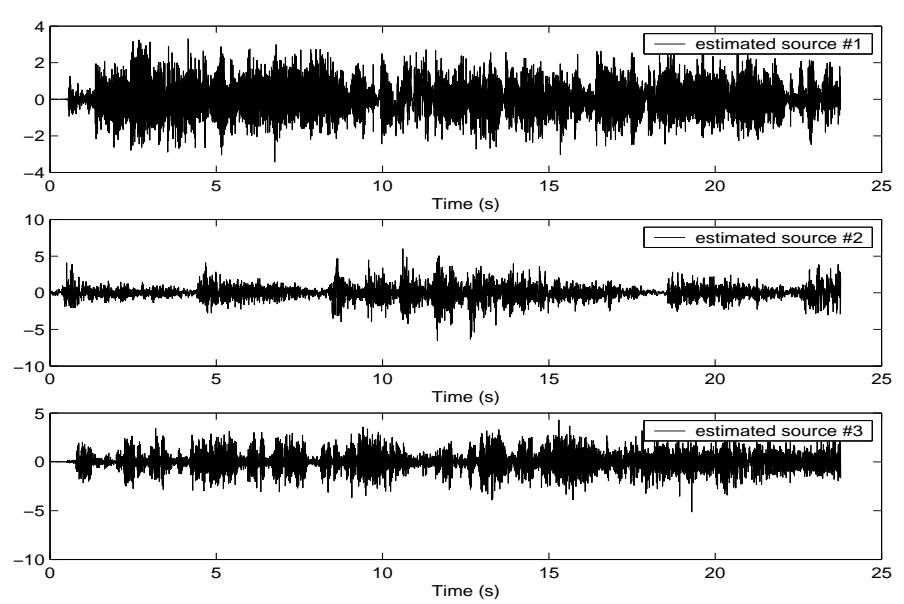

Fig. 2. Estimated sources
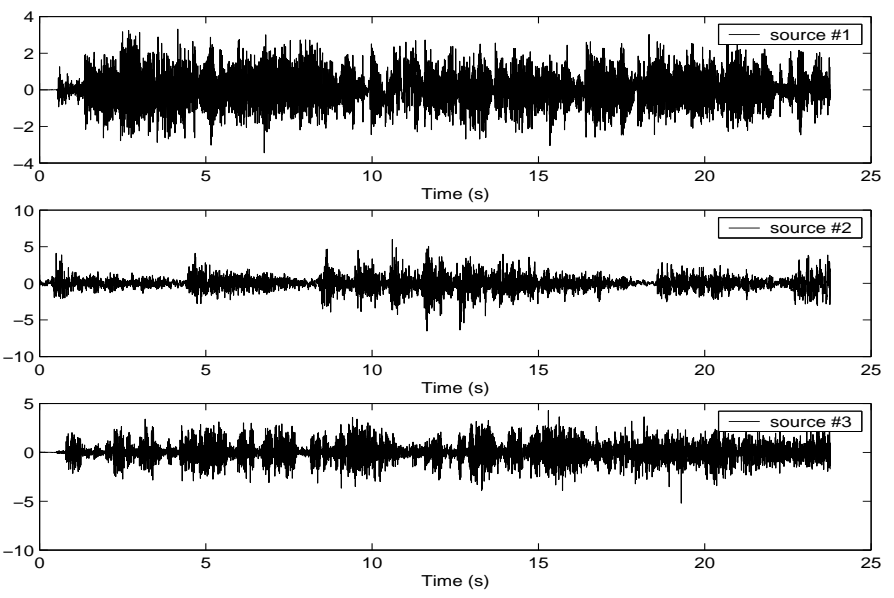

Fig. 3. Original sources

The mean square errors (MSE) between the estimated sources and the original ones and the corresponding Signal to Interference Ratio (SIR) are presented in the Table 1.

\begin{tabular}{|c|c|c|c|}
\hline & source \#1 & source \#2 & source \#3 \\
\hline MSE & 0.0141 & 0.0155 & 0.0164 \\
\hline SIR & $41 \mathrm{~dB}$ & $44 \mathrm{~dB}$ & $36 \mathrm{~dB}$ \\
\hline
\end{tabular}

Table 1. Performances

\section{CONCLUSION}

This paper present a novel iterative method for blind source separation from delayed mixtures. The delayed mixtures are approached by instantaneous mixtures of the observation derivatives. This approach uses only second-order statistics and needs no more mixtures than sources. The algorithm is based on the joint diagonalization of a set of spatial covariance matrices but the non square separating matrix is constrained to hold a theoretical structure at each iteration. A numerical simulation shows the efficiency of the approach.

\section{APPENDIX A}

From $\mathbf{M}^{f}(\nu)$ To S In the 2-ObSERVATIONS CASE.

Now, we have the following spectral mixing matrix

$$
\mathbf{M}^{f}(\nu)=\left[\begin{array}{cc}
1 & 1 \\
c_{21} e^{-\jmath 2 \pi \nu \tau_{21}} & c_{22} e^{-\jmath 2 \pi \nu \tau_{22}}
\end{array}\right] .
$$

In this case (6) leads to the 2 following filtered sources:

$$
\begin{aligned}
& x_{1}^{h}(t)=c_{22} y_{1}\left(t-\tau_{22}\right)-y_{2}(t), \\
& x_{2}^{h}(t)=-c_{21} y_{1}\left(t-\tau_{21}\right)+y_{2}(t),
\end{aligned}
$$

where $x_{k}^{h}(t)=x_{k}\left(t-\tau_{22}\right)-x_{k}\left(t-\tau_{21}\right), k \in[1,2]$. 
With $\mathbf{x}^{h}(t)=\left[x_{1}^{h}, x_{2}^{h}\right]^{T}$, and $\tilde{\mathbf{y}}(t)=\left[y_{1}, \dot{y}_{1}, \ddot{y}_{1}, \ldots, y_{1}^{(P)}, y_{2}\right]^{T}$, the $(2 \times P+2)$ unmixing matrix of equation (7) becomes:

$$
\mathbf{S}=\left[\begin{array}{ccccc}
c_{22} & -c_{22} \tau_{22} & \ldots & c_{22} \frac{\left(-\tau_{22}\right)^{P}}{P !} & -1 \\
-c_{21} & c_{22} \tau_{21} & \ldots & -c_{21} \frac{\left(-\tau_{21}\right)^{P}}{P !} & 1
\end{array}\right]
$$

\section{APPENDIX B}

From $\mathbf{S}$ TO $c_{i j}, \tau_{i j}$ IN THE 2-OBSERVATIONS CASE.

Here, we have the following separating matrix

$$
\mathbf{S}=\left[\begin{array}{lllll}
s_{11} & s_{12} & \ldots & s_{1 P} & s_{1 P+1} \\
s_{21} & s_{22} & \ldots & s_{2 P} & s_{2 P+1}
\end{array}\right]
$$

one has the $(2 \times 2)$ spectral separating matrix

$$
\mathbf{S}^{f}(\nu)=\left[\begin{array}{ll}
s_{11}^{f}(\nu) & s_{12}^{f}(\nu) \\
s_{21}^{f}(\nu) & s_{22}^{f}(\nu)
\end{array}\right]
$$

where the entries of $\mathbf{S}^{f}(\nu)$ are polynomials in $\nu$ :

$$
\begin{array}{rlrl}
s_{11}^{f}(\nu) & = & \sum_{k=1}^{P}(2 j \pi \nu)^{k} s_{1 k}, \\
s_{12}^{f}(\nu) & = & s_{1 P+1}, \\
s_{21}^{f}(\nu) & = & \sum_{k=1}^{P}(2 j \pi \nu)^{k} s_{2 k}, \\
s_{22}^{f}(\nu) & = & & s_{2 P+1} .
\end{array}
$$

The inverse matrix of $\mathbf{S}^{f}(\nu)$ is also a matrix of polynomials:

$$
\left[\mathbf{S}^{f}(\nu)\right]^{-1}=\frac{1}{\operatorname{det} \mathbf{S}^{f}(\nu)}\left[\begin{array}{ll}
p_{11}(\nu) & p_{12}(\nu) \\
p_{21}(\nu) & p_{22}(\nu)
\end{array}\right] .
$$

An approximation of the spectral mixing matrix $\mathbf{M}^{f}(\nu)$ is obtained normalizing each column of the previous matrix by $p_{1 k}(\nu)$

$$
\mathbf{M}^{f}(\nu) \approx\left[\begin{array}{cc}
1 & 1 \\
p_{21}(\nu) / p_{11}(\nu) & p_{22}(\nu) / p_{12}(\nu)
\end{array}\right] .
$$

The entries of the second line of this matrix can be developed in power series expansion in $\nu$ :

$$
\begin{array}{ll}
p_{21}(\nu) / p_{11}(\nu)= & q_{0}+q_{1} \nu+O^{2}(\nu), \\
p_{22}(\nu) / p_{12}(\nu)= & r_{0}+r_{1} \nu+O^{2}(\nu) .
\end{array}
$$

Identifying these expansions with the Taylor-series expansion of the entries of $\mathbf{M}^{f}(\nu)$ (see 9) we find:

$$
\begin{aligned}
c_{21} & =q_{0}, \\
\tau_{21} & =-\frac{1}{j 2 \pi} \frac{q_{1}}{q_{0}}, \\
c_{22} & =r_{0}, \\
\tau_{22} & =-\frac{1}{j 2 \pi} \frac{r_{1}}{r_{0}} .
\end{aligned}
$$

\section{REFERENCES}

[1] G. Chabriel and J.Barrère, Blind Identification of Slightly Delayed Mixtures, in Proc. of the Tenth IEEE Workshop on Statistical Signal and Array Processing, pp. 319-323, August 14-16, 2000.

[2] J. Barrère and G. Chabriel, A Compact Sensor Array for Blind Separation of Sources, IEEE Trans. on Circuits and Systems, Part I, Vol. 49, No 5, pp. 565-574, May 2002.

[3] G. Chabriel and J. Barrère, An Instantaneous Formulation of Mixtures for Blind Separation of Propagating Waves, IEEE Trans. on Signal Processing, Vol. 54, No. 1, pp.4958 Jan. 2006.

[4] G. Cauwenberghs, M. Stanacevic and G. Zweig, Blind Broadband Source Localization and Separation in Miniature Sensor Arrays in Proc. of IEEE Int. Symp. Circuits and Systems (ISCAS'2001), Vol. 3, pp. 193-196, Sydney, Australia, 2001.

[5] A. Yeredor, Blind Source Separation with Pure Delay Mixtures, in Proc. $2^{\text {nd }}$ International Symposium on Independent Component Analysis and Blind Signal Separation (ICA’2001), pp. 691-696, December 2001, San diego - California, USA.

[6] M. Stanacevic and G. Cauwenberghs, Micropower Gradient Flow VLSI Acoustic Localizer in IEEE Transactions on Circuits and Systems I : Regular Papers, to appear, 2005.

[7] S. Dégerine, Sur la diagonalisation conjointe approchée par un critère des moindres carrés, in Proc. of the $18^{e}$ Colloque GRETSI sur le traitement du signal et des images, pp. 311-314, Toulouse 2001.

[8] Serge Dégerine and Elimane Kane, A new non-orthogonal approximate joint diagonalization algorithm for blind source separation, in http://www-lmc.imag.fr/lmcsms/Serge.Degerine/Publications/LSBr.pdf 